\title{
Determination of majority carrier capture rates via Deep Level Transient Spectroscopy
}

\author{
J. Lauwaert, ${ }^{1, *}$ S. Khelifi, ${ }^{1}$ and H. Vrielinck ${ }^{2}$ \\ 1 Department of Electronics and Information Systems, \\ Ghent University, St.-Pietersnieuwstraat 41, 9000 Gent, Belgium \\ 2 Department of Solid State Sciences, Ghent University, \\ Krijgslaan 281-S1, 9000 Gent, Belgium
}

(Dated: October 27, 2015)

\begin{abstract}
In Deep Level Transient Spectroscopy (DLTS) experiments the majority carrier capture rate is often determined by observing the growth of the signal amplitude as a function of filling pulse duration at constant temperature. The analysis of such experiments is complicated by the phenomenon of slow capture: carrier capture by defects in the Debye tail of the depletion layer at the pulse voltage. We review here three approaches for analyzing isothermal pulse duration variation DLTS experiments that have been described or at least have been frequently used in literature. These methods are compared for their ability to correctly extract capture rates from simulated data as well as from actual experimental data for the $\mathrm{Fe}^{-/ 2-}$ level in crystalline germanium. Finally, we tested the performance of the three methods for analysis of DLTS signals that experience a delayed growth, modeled by an additional time constant in the system.

PACS numbers:
\end{abstract}

*Electronic address: Johan. Lauwaert@UGent. be 


\section{INTRODUCTION}

In the four decades since its introduction in the seminal paper of Lang [1], Deep-Level Transient Spectroscopy (DLTS) has grown out to the standard technique for characterization of point defects that introduce deep levels in the band gap of semiconductors. In classical DLTS transients $\left(\Delta C \exp \left(\frac{-t}{\tau}\right)\right)$ in the high-frequency reverse capacitance $\left(C_{r}\right)$ of a (Schottky or p-n) diode depletion layer are measured after application of voltage pulses. These arise from slow emission of electrons (rate $e_{n}$ ) or holes (rate $e_{p}$ ) by defects in the depletion layer that change their charge state after returning from the pulse voltage $\left(V_{p}\right)$ back to the reverse voltage $\left(V_{r}\right)$, because they had captured a carrier ( rate $c_{n} n=\sigma_{n} v_{t h} n$ for electrons or $c_{p} p=\sigma_{p} v_{t h} p$ for holes) during the pulse. Transients are measured as a function of temperature $(T)$, the pulse voltage parameters $V_{r}, V_{p}$ and filling pulse time $\left(t_{p}\right)$. Figure 1a shows an example of the capacitance transients in case of majority carrier capture and emission at a fixed temperature. They may be analyzed by various filter functions [2] with characteristic window times $\left(t_{W}\right)$ or by Laplace transformation[3]. Subsequent data analysis (in principle) allows to determine energy level positions in the bandgap and charge state/transition assignments, carrier capture cross-sections and activation barriers $\left(\Delta E_{\sigma}\right)$ therein, and defect concentration (profiles), in principle both for majority and for minority carriers. This analysis is based on the theory of DLTS for ideal diodes with low concentrations of deep-level defects, which is well-established and covered by several textbooks. When applying DLTS to real devices, however, several deviations may occur that can influence the interpretation of the spectra.[5-7] This paper focuses on one particular problem in DLTS: determining the majority carrier capture cross-section $\sigma$.

In an Arrhenius analysis of the temperature dependence of the carrier emission rate measured by DLTS, the pre-exponential factor already provides information about $\sigma$. [4] Careful thermodynamic analysis demonstrates, however, that entropy changes in the emission process need to be taken properly into account for obtaining accurate $\sigma$ values. Observation of the growth of the transient amplitude as a function of pulse time $\left(\Delta C\left(t_{p}\right)\right)$ at constant $T$ presents an independent experimental method for determining $\sigma$, that does not require information on entropy. This method was already introduced by Lang[1]. It is limited by the minimum pulse duration (in practice $\sim 10^{-8} \mathrm{~s}$ ) of the setup and can therefore only be applied to carrier trap levels with (moderate and) small $\sigma$ in devices with moderate free carrier con- 

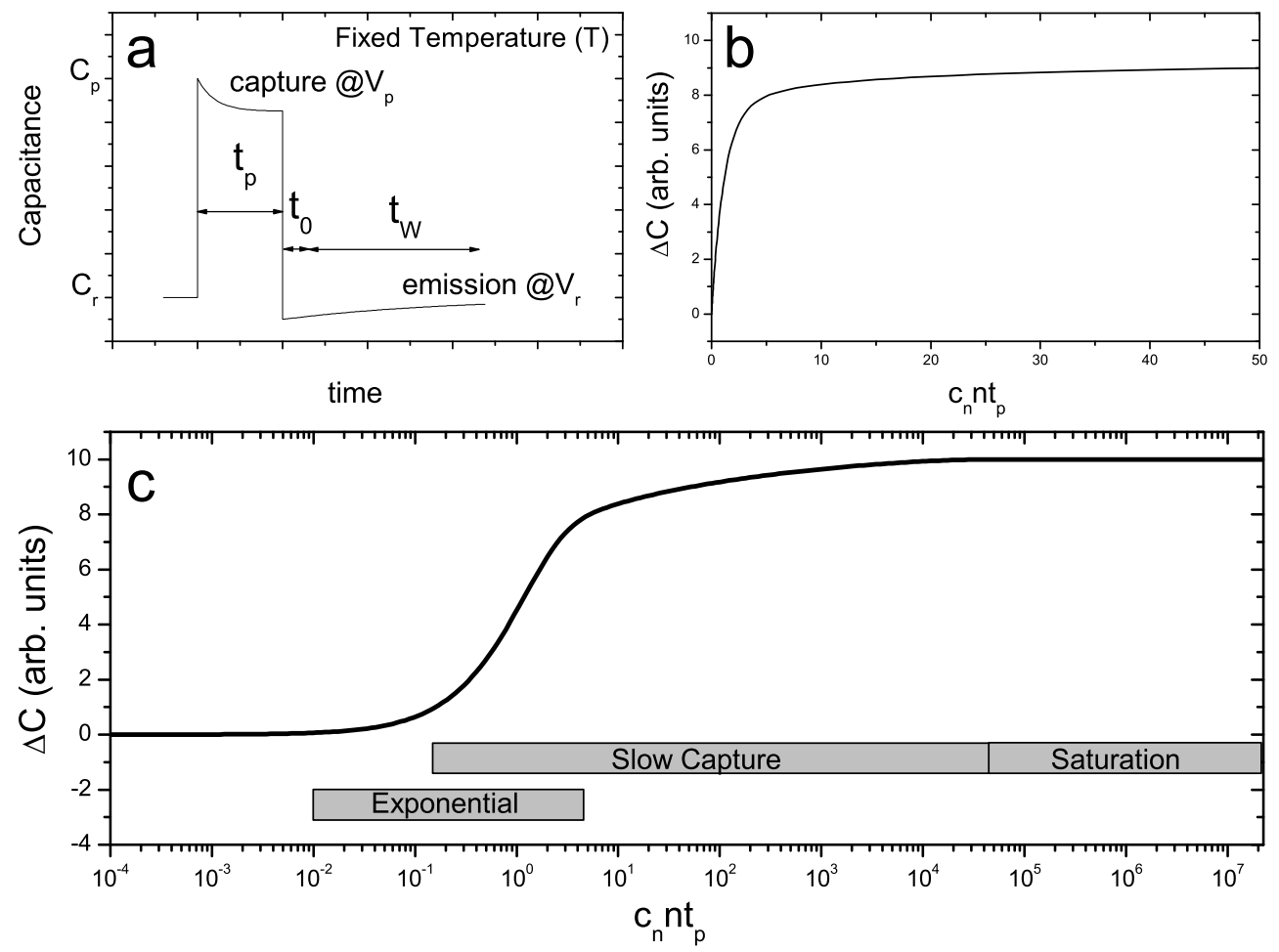

FIG. 1: a) pulse sequence applied to determine majority carrier trap parameters, defining the experimental parameters of the pulse. b) typical (simulated) $\Delta C\left(t_{p}\right)$ curve in linear and c) the same curve in exponential pulse time scale. In the abscissa, the dimensionless parameter $c_{n} n t_{p}$ (capture rate $\times$ pulse time) is presented

centrations (e.g. $c_{n} n<1 \times 10^{8} \mathrm{~s}^{-1}$ for $n=1 \times 10^{14} \mathrm{~cm}^{-3}$ implies $\sigma_{n}<1 \times 10^{-13} \mathrm{~cm}^{2}$ ). Basic theory predicts a saturating exponential growth for $\Delta C\left(t_{p}\right)$. This is seen in Fig. 1b,c where simulated data are presented (for electrons) as a function of the dimensionless parameter $c_{n} n t_{p}$, at least for sufficiently small $t_{p}$ values (up to $c_{n} n t_{p} \approx 2$ ). However, before actually saturating, a regime of slower growth of $\Delta C\left(t_{p}\right)$ is entered, generally referred to as slow capture. It arises from carrier capture by defects in the Debye tail of the depletion layer (at $V_{p}$ ), where the free carrier concentration is rapidly decreasing. This effect complicates the analysis of $\Delta C\left(t_{p}\right)$ and for reliable extraction of $\sigma$ it should somehow be taken into account. Pons found a closed expression for the amplitude of the capacitance transient as a function 
of the pulse length[8].

$$
\frac{\Delta C\left(t_{p}\right)}{C_{r}}=-\frac{1}{W_{r}^{2} n} \int_{0}^{+\infty} N_{T}(x) \Delta f\left(x, t_{p}\right) x d x
$$

With $C_{r}$ and $W_{r}$ the quiescent capacitance and depletion width at $V_{r}, x$ the distance from the junction, $N_{T}(x)$ the trap concentration, $n$ the free carrier concentration, and $\Delta f$ the change in fractional occupation of the trap levels. In order to derive $\sigma$ from this expression by fitting, it needs to be approximated. Early proposals for such approximations, based on the depletion approximation, did not properly take into account re-emission during capture and led to divergence for $\Delta C\left(t_{p} \rightarrow+\infty\right)$. Lauwaert et al. worked out an analytical approximation, that does not suffer from these drawbacks[9]. For accurate estimates of $\sigma$, the $\Delta C\left(t_{p}\right)$ curve should comprise a range where the effect of slow capture is not dominant. This presents the main restriction on the use of this method, which has been successfully applied in determining $\sigma$ for various transition metal related acceptor levels in n-type Ge[11-14], by fitting $\Delta C\left(t_{p}\right)$ in an extensive $t_{p}$ range. Here we consider this analytical approximation as a reference for comparison with two conceptually simpler, experiment-based approaches for determining $\sigma$ from $\Delta C\left(t_{p}\right)$ curves.

The simplest way of dealing with slow capture is excluding the long pulse $t_{p}$ range, where slow capture has the most prominent effect, from the analysis. Evidently, decreasing the number of data points in the analysis leads to results with lower accuracy and narrows down the range of capture rates that can be analyzed. By choosing the experimental parameters $V_{r}$ and $V_{p}$ wisely - maximizing the ratio between the contribution from normal fast capture and that from slow capture, one may still try and extend the application range of this method somewhat. In the limiting case of an infinitely wide depletion layer at $V_{r}$ $\left(V_{r} \rightarrow-\infty\right)$, and $\left(C_{r} \rightarrow 0\right)$, the contribution from slow capture should, indeed, become negligible. We label this approach here as Method 1.

Exactly in the latter consideration of the limiting case lies the key to a second possible approach. Keeping $V_{p}$ constant, one may record $\Delta C\left(t_{p}\right)$ for increasing values of $V_{r}$, from these data extract $\sigma$ as a function of $C_{r}$ by considering only the part of the curves where $\Delta C\left(t_{p}\right)$ is dominated by normal capture, and extrapolate the resulting $\sigma\left(C_{r}\right)$ curve to $C_{r} \rightarrow 0$. Segers et al. recently proposed this approach for the analysis of quenched-in defects in p-type Ge[16]. Here, we label it as Method 2.

In this paper we compare these two simplified approaches with the analytical approximation 
by Lauwaert et al.[9] which we label as the Analytical Method. In Section 2 we explain how these three methods relate to the general theory of Pons and how they can be used in the analysis of $\Delta C\left(t_{p}\right)$ curves. In Section 3 the three methods are compared by analyzing data generated through simulations and on an actual experimental example: the $\mathrm{Fe}^{-/ 2-}$ level in n-type Ge. We present analyses for the capture rate $c_{n} n$ or $c_{p} p$, since these are the properties directly extracted from experiments. At a given temperature $\sigma$ is then easily calculated when the carrier concentration is determined from the $\mathrm{C}-\mathrm{V}$ curve. Finally, Section 4 focuses on a peculiar feature observed in the $\Delta C\left(t_{p}\right)$ for quenched-in defects in p-type Ge: the curves exhibit an initial $\mathrm{t}_{\mathrm{p}}$-range where $\Delta C\left(t_{p}\right)$ remains zero, or in other words, a delayed growth of the signal. Since this range decreases when increasing $V_{r}$, just like the slow capture contribution as discussed by Pons[8], in Ref. [16] this effect was also attributed to slow capture. We show that in this case fitting the data via Method 2 is the best option for obtaining reliable estimates of capture rates and cross-sections.

\section{THEORY}

If one merely wants to calculate the trap concentration $N_{T}$ from $\Delta C$, one assumes that for long pulse times $t_{p}$ the DLTS signal is saturated and Eq. (1) is simplified by limiting

the borders of the integral to the region where $\Delta f(x)>\frac{1}{2}$. Then Eq. (1) reduces to the conventional 'pulse correction factor' [10]:

$$
\Delta C=\frac{1}{2} \frac{N_{T}}{n W_{r}^{2}}\left(\left(W_{r}-L_{r}\right)^{2}-\left(W_{p}-L_{p}\right)^{2}\right) C_{r}
$$

with $W_{r}$ and $W_{p}$ the depletion widths at $V_{r}$ and $V_{p}$, respectively, and $L_{r}=L_{p}$ the distance from the edge of the depletion layer to the position $x$ where $\Delta f(x)=1 / 2$. For shorter pulses $t_{p}$ two effects contribute to the growth of $\Delta C$. First, within this short pulse time only a fraction of the traps can change their occupancy and $\Delta f$ becomes $t_{p}$ dependent. Second, due to the slow capture in the Debye tails the position $L_{p}$ (relative to $W_{p}$ ) for which $\Delta f=1 / 2$ also becomes $t_{p}$ dependent. In Ref [9] this $t_{p}$ dependence is calculated analytically. Including these two prerequisites in the pulse correction factor (2) leads to the following analytical approximation for $\Delta C\left(t_{p}\right)$

$$
\Delta C\left(t_{p}\right)=\frac{1}{2} \frac{N_{T} \Delta f_{\max }\left(t_{p}\right)}{n W_{r}^{2}}\left(\left(W_{r}-L_{r}\right)^{2}-\left(W_{p}-L_{p}\left(t_{p}\right)\right)^{2}\right) C_{r}
$$




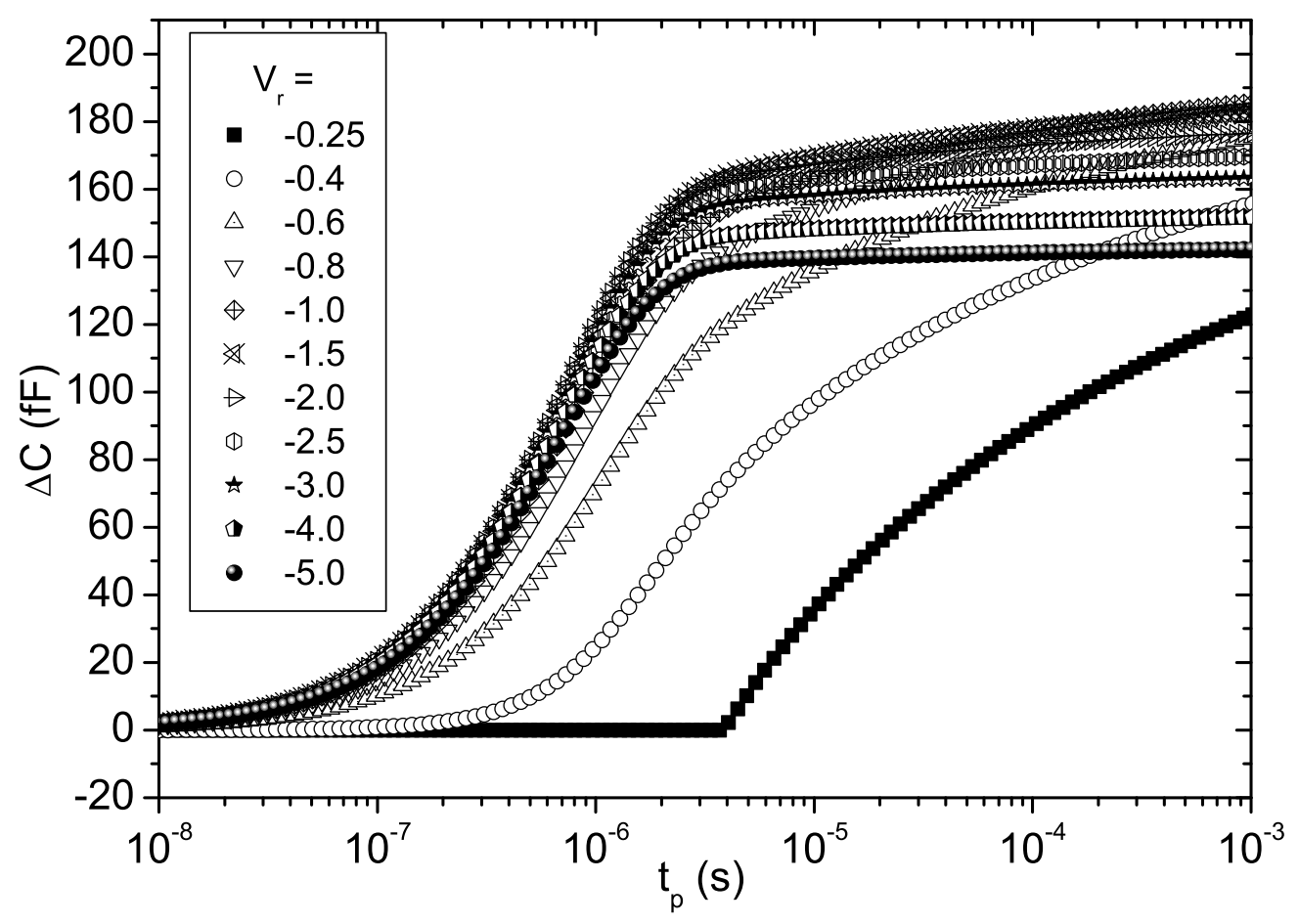

FIG. 2: DLTS-signal amplitude as a function of filling pulse duration for different reverse voltages calculated using the analytical approximation and the parameters shown in table I.

with $\Delta f_{\max }\left(t_{p}\right)$ the maximum of the function $\Delta f(x)$. Figure 2 shows an example of an analytical calculation with the parameters in table I. For short $t_{p}$ and small pulse heights $W_{r}-L_{r}$ can be smaller than $W_{p}-L_{p}$. Within this analytical approximation this would result in a negative signal. In order to correct for this only positive results are included in the fitting and the negative values are set to zero as is seen in the curve for $V_{r}=-0.25 \mathrm{~V}$ in Fig. 2. The threshold for the minimum pulse height as a function of $t_{p}$ was calculated theoretically by Pons [8]. Eq. 3 may be further separated into a term that describes the normal exponential capture and a second due to slow capture. Hence, we can rewrite Eq. 3 as

$$
\Delta C\left(t_{p}\right)=\frac{1}{2} \frac{N_{T} \Delta f_{\max }}{n_{0} W_{r}^{2}}\left(\left(W_{r}-L_{r}\right)^{2}-\left(W_{p}\right)^{2}\right) C_{r}+\alpha\left(t_{p}\right) C_{r}^{3}
$$

with

$$
\alpha\left(t_{p}\right)=-\frac{L_{p}^{2}-2 W_{p} L_{p}}{\epsilon A} \frac{N_{T}}{2 n_{0}} \Delta f_{\max }
$$




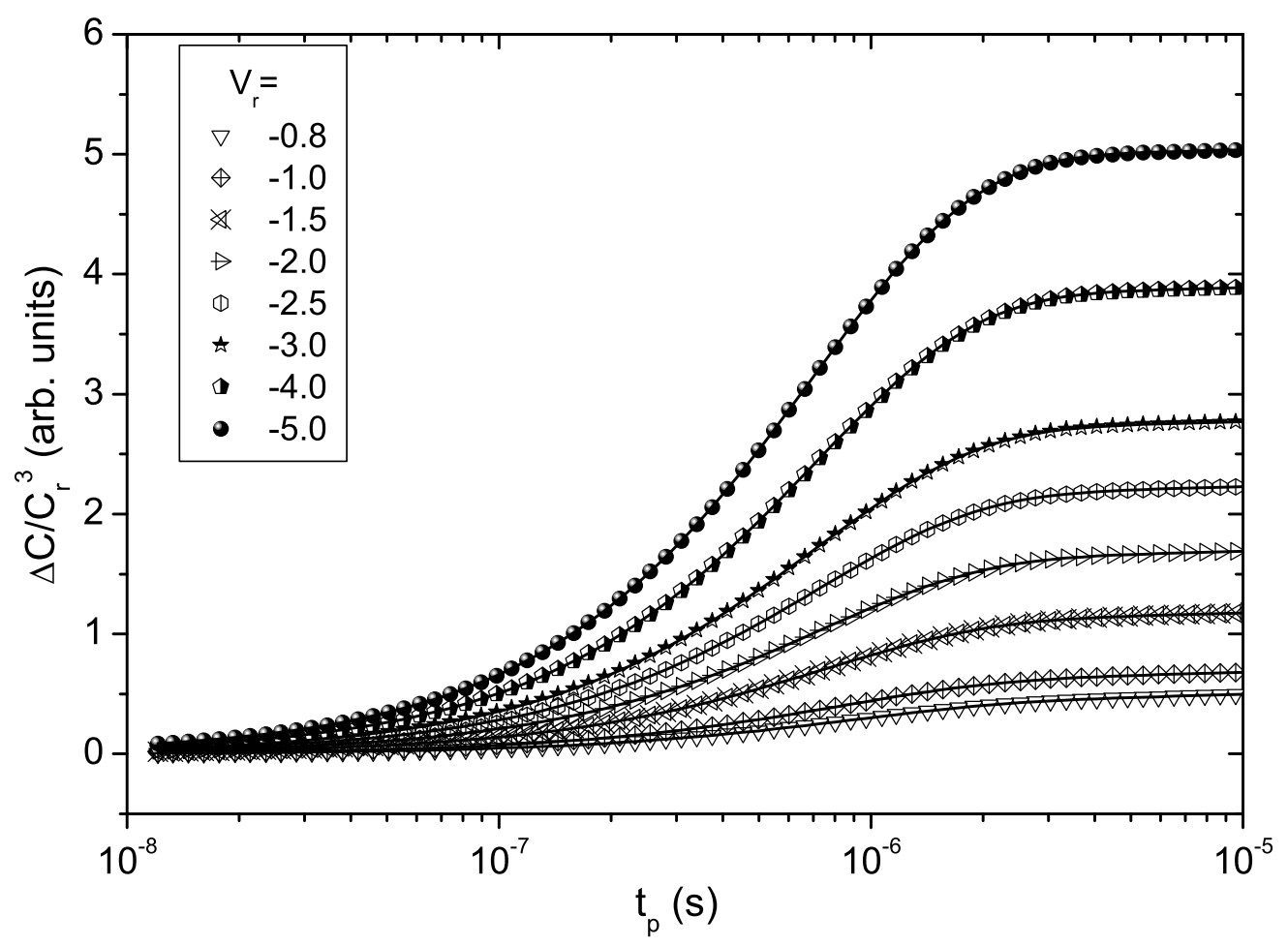

FIG. 3: DLTS-signal amplitude as a function of filling pulse duration for different reverse voltages calculated using the analytic approximation divided by $C_{r}^{3}$.

Since $\alpha\left(t_{p}\right)$ is independent of $C_{r}$, the second term, including the contribution of slow capture, is proportional to $C_{r}^{3}$. This is demonstrated in Fig. 3, where the data of Fig. 2 are divided by $C_{r}^{3}$. One can clearly see that the part of the curves that is dominated by slow capture $\left(t_{p}>10^{-6} s\right)$ is the same for all $V_{r}$ values, and hence is independent of $C_{r}$. This will allow to fit $\Delta C / C_{r}^{3}$ curves for different reverse biases $V_{r}$ simultaneously as is mentioned later in this section.

In a first approach to analyzing the $\Delta C\left(t_{p}\right)$ curves - Method 1 - the long pulse duration range, where slow capture becomes prominent, is avoided. Method 1 thus neglects the $t_{p}$ dependence of $L_{p}$ and therefore assumes that $\Delta C\left(t_{p}\right)$ is proportional to $\Delta f_{\max }$

$$
\Delta f_{\text {max }}=\left(1-\exp \left(-t_{p}\left(c_{n} n+e_{n}\right)\right)\right)\left(\frac{c_{n} n}{c_{n} n+e_{n}}\right)
$$

If the concentration of injected charge carriers is high enough, $c_{n} n>e_{n}$, and this fractional occupation is further simplified to: 


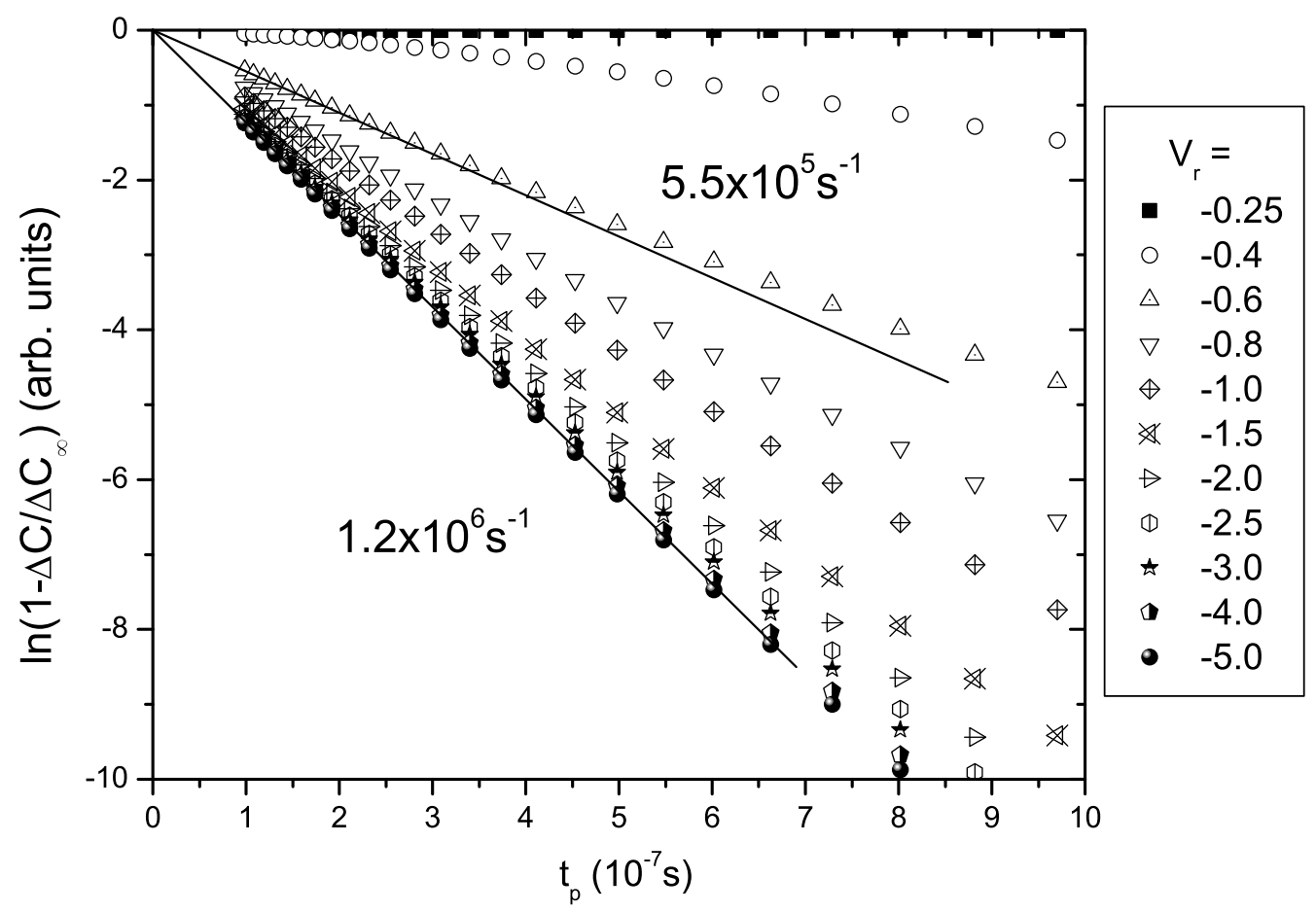

FIG. 4: $\ln \left(1-\frac{\Delta C\left(t_{p}\right)}{\Delta C_{\infty}}\right)$ for the calculated DLTS-signal amplitudes as a function of filling pulse duration.

$$
\Delta f \approx\left(1-\exp \left(-t_{p} c_{n} n\right)\right)
$$

Indeed in the neutral region where the normal capture occurs this is well justified. Since for a uniform injection of carriers and a uniform deep trap concentration Eq. (1) is proportional to this fractional occupation, it is possible to approximate the DLTS-signal as:

$$
\frac{\Delta C\left(t_{p}\right)}{\Delta C_{\infty}}=\left(1-\exp \left(-c_{n} n t_{p}\right)\right)
$$

For such dependence $\Delta C\left(t_{p}\right)$ the range in which the signal grows from 1 to $99 \%$ of its maximum value corresponds with $0.01<c_{n} n t_{p}<4.6$. This range is indicated with Exponential in Fig. 1. On the other hand, from the analytical approximations for $L_{p}$ suggested in Ref. [9], we can expect a significant effect from the slow capture for $c_{n} n t_{p}>0.15$. This range is labeled Slow Capture in Fig. 1. It is therefore expected that Eq. (8) only is a good 

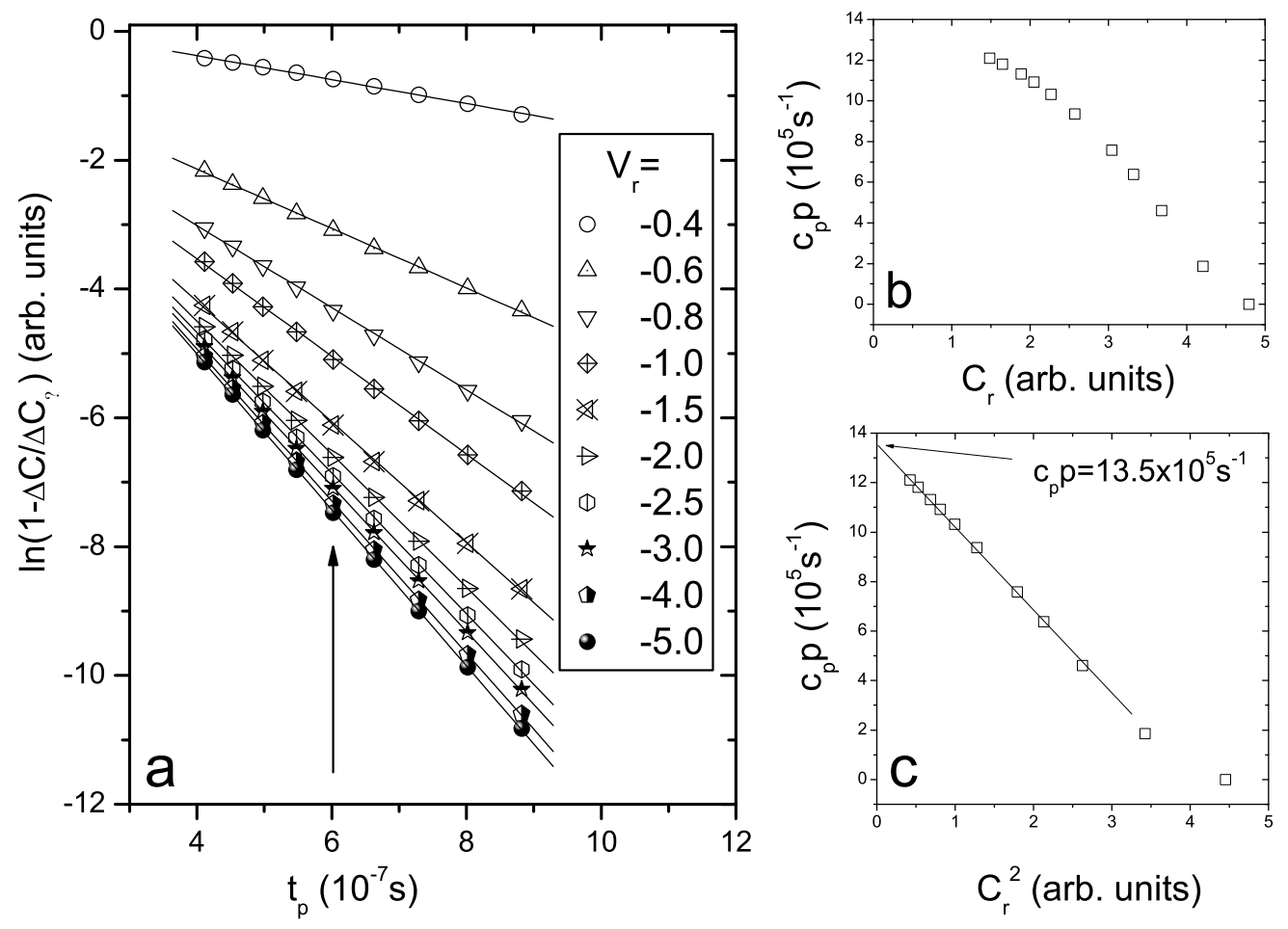

FIG. 5: (a) Determination of the derivation at $t_{p}=6 \times 10^{-7} s$ using Eq. (9) (b) Linear extrapolation of the slope to $C_{r}=0$ (c) Quadratic extrapolation of the slope to $C_{r}=0$

approximation for small $t_{p}$. In absence of slow capture effects,

$$
\ln \left(1-\frac{\Delta C\left(t_{p}\right)}{\Delta C_{\infty}}\right)
$$

is proportional to $t_{p}$, with $c_{n} n$ as proportionality constant. In anticipation of slow capture effects, the best estimate of $c_{n} n$ from this dependence is obtained from the slope of a straight line through the origin and the point at the smallest $t_{p}$ for which a change in $\Delta C$ is observed. This method is illustrated in Fig. 4 for the simulations in Figs. 2 and 3 with parameters shown in Table I, further assuming that only the data for $t_{p}>10^{-7} s$ are experimentally available.

Method 2 for analyzing $\Delta C\left(t_{p}\right)$ curves uses the first derivative of $\Delta C\left(t_{p}\right)$ at a certain $t_{p}$ point to estimate $c_{n} n$ as a function of $C_{r}$. Figure 5 a shows how the slope at $t_{p}=6 \times 10^{-7} s$ is determined numerically using adjacent points. Based on a further approximation of Eq. 
(4), we see that the capture rate can be derived from this slope:

$$
\frac{1}{\Delta C_{\infty}-\Delta C\left(t_{p}\right)} \frac{d \Delta C\left(t_{p}\right)}{d t_{p}}=c_{n} n+2 \frac{d \alpha\left(t_{p}\right)}{d t_{p}} \frac{n_{0}}{N_{T}} C_{r}^{2}
$$

Where the denominator $\Delta C_{\infty}-\Delta C\left(t_{p}\right)$ is approximated in a similar way as in Method 1 , i.e. neglecting the term proportional to $\alpha\left(t_{p}\right)$. For the second term Eq. (4) is simplified even further. For large $W_{r}, L_{r} \ll W_{r}$ and $W_{p} \ll W_{r}$ which makes that the denominator can be simplified to:

$$
\left(1-\frac{L_{r}}{W_{r}}\right)^{2}-\left(\frac{W_{p}}{W_{r}}\right)^{2} \approx 1
$$

From Eq. (10) it follows that for large $W_{r}$ - or for $C_{r} \rightarrow 0$ - the perturbation by slow capture is purely quadratic in $C_{r}$. This is the basis for the second method. The slope of $\Delta C\left(t_{p}\right)$ curve at a chosen $t_{p}$ point (that in principle may differ for $\Delta C\left(t_{p}\right)$ curves recorded at different $V_{r}$ ) is determined as a function of $C_{r}^{2}$ (by varying $V_{r}$ at fixed $V_{p}$ ) and this curve is linearly extrapolated to $C_{r}^{2} \rightarrow 0$. This is shown in Fig. 5b. Because this method only makes use of the first derivative of Eq. (9) in one point we may expect that the accuracy depends on the choice of $t_{p}$. Like Method 1, Method 2 can only yield reasonable estimates of capture rates (and cross-sections) when the contribution of slow capture is small relative to that of normal capture. For the last two points in Fig. 5b (at large $C_{r}^{2}$ value, or small $\left|V_{r}\right|$ ), the contribution of slow capture at the chosen $t_{p}$ value is already too large to perfectly follow the $C_{r}^{2}$ dependency. Including these points in the extrapolation would lower the estimate of the capture rate. Method 2, however, also presents an important advantage with respect to Method 1, more particularly when the exponential growth regime of the $\Delta C\left(t_{p}\right)$ curve is delayed, e.g. an additional time constant in the device (see Section IV), which will necessarily lead to underestimates of the capture rate. Method 2 offers freedom in the choice of the $t_{p}$ point where the $\frac{d \Delta C}{d t_{p}}$ is calculated, and in such case the maximum of the derivative is expected to yield the best results.

The third, Analytical Method has been described extensively in Ref [9]. A small addition of this work is that the $\Delta C\left(t_{p}\right)$ curves recorded at increasing $\left|V_{r}\right|$ are all fitted simultaneously with the analytical model. This is possible because the parameters that include the slow capture are proportional to $C_{r}^{3}$ and the capture rate is independent of $V_{r}$. Therefore, including an extra curve in the fitting for a different $V_{r}$ but with same $V_{p}$ for all curves, induces 
TABLE I: Parameters used for the calculation of the analytical curves shown in Fig. 2.

\begin{tabular}{cc} 
Parameter & Value \\
\hline$t_{0}$ & $1.283 \mathrm{~ms}$ \\
$t_{W}$ & $6.912 \mathrm{~ms}$ \\
$p$ & $2.0 \times 10^{14} \mathrm{~cm}^{-3}$ \\
$N_{T}$ & $1.2 \times 10^{12} \mathrm{~cm}^{-3}$ \\
$\sigma$ & $5.6 \times 10^{-14} \mathrm{~cm}^{2}$ \\
$e_{p}$ & $72 \mathrm{~s}^{-1}$
\end{tabular}

only one extra parameter $a_{3}$ following the nomenclature of Lauwaert et al. [9]:

$$
a_{3}=\frac{1}{n W_{r}} \int_{W_{p}}^{W_{r}-L_{r}} N_{T}(x) x d x
$$

\section{COMPARISON OF THE $\Delta C\left(t_{p}\right)$ ANALYSIS METHODS}

Figure 6 forms the basis for the comparison of the different analysis methods discussed in Section 3 with respect to their accuracy in extracting the majority (holes) carrier capture cross-section, expressed as the capture rate $c_{p} p$. The ordinate value of a data point in this graph represents the $c_{p} p$ result obtained by analyzing via Methods 1 and 2 the simulated data in Figs. 2 and 3, generated with the analytical model using the parameters listed in Table I. The abscissa value represents the minimum $t_{p}$ value for which data are available. Furthermore, analysis results are presented for data at or up to $V_{r}=-2 \mathrm{~V}$ and $V_{r}=-5 \mathrm{~V}$. Obviously, the capture rate used in the simulation is found back when analyzing the data with the Analytical Model. This is represented as a horizontal solid line in the figure, which should here represent the ideal value that Methods 1 and 2 should approach. The $t_{p}$ range where the normal and the slow capture contributions to $\Delta C$ vary from $1 \%$ to about $99 \%$ of their maximum are indicated with the grey bars: they overlap by about 1.5 decade. Both Method 1 and Method 2 lead to reasonable estimates for the capture rate (10-20\% deviating from the value used in the simulations) if data in a significant fraction of the exponential growth regime are available. In such conditions (at small abscissa values), thus on the left hand side of the graph, the fitting result hardly depends on the $t_{p}$ point where 
the $\Delta C\left(t_{p}\right)$ curve starts. However, if data are only available from $t_{p}$ values onwards where the growth of $\Delta C$ is dominated by slow capture, the accuracy of the fitting result rapidly decreases. Thus, both simplified methods exhibit a transition region between fairly accurate and unreliable results for $1 \mu s<t_{p}<2 \mu s$. Increasing the $\sigma$ value shifts both the fast and the slow capture regime to lower $t_{p}$ values, and consequently also this transition region. We may further note that increasing $\left|V_{r}\right|$ has, as expected, a positive influence on the fitting result for both simplified methods: it leads to more accurate fitting values if data are available at sufficiently short pulses. Unfortunately, it does not shift the transition region much, or in other words, it does not extend the range of measurable capture cross-sections significantly. Since Method 2 applies extrapolation of capture rate estimations, which increase as $C_{r}$ decreases $\left(\left|V_{r}\right|\right.$ increases) one may anticipate that the analysis following this method will lead to higher results than when applying Method 1. This is nicely illustrated in Fig. 6. Because of the neglect of slow capture, Method 1 should always underestimate the actual capture rates. Method 2 may lead to overestimation of the capture rate, but only when data are available from sufficiently small pulse durations.

Fitting the data with the model used for simulating them, obviously leads to accurate results of capture rates. Restricting the available $t_{p}$ range on the short pulse side, however, still leads to an increase in the fitting error, which is smaller if data are used up to $V_{r}=-5 \mathrm{~V}$ (dashed lines in Fig. 6 indicate the borders of the $95 \%$ confidence interval for the fitting result) than when the maximum $\left|V_{r}\right|$ value is restricted to $2 \mathrm{~V}$ (dotted lines). Fig. 6 thus indicates that in comparison with methods 1 and 2, the Analytical Method shifts the $t_{p}$ range where the fitting becomes unreliable by about one decade towards longer pulse lengths. Hence, fitting with the analytical model does extend the range of measurable capture rates (cross-sections).

As a final point of this section, we apply fitting methods 1 and 2, as well as the Analytical Method to $\Delta C\left(t_{p}\right)$ curves measured at $155 \mathrm{~K}$ for the DLTS peak associated with the $\mathrm{Fe} e^{-/ 2-}$ level in n-type Ge. Previous DLTS analyses of the electron trapping parameters for $\mathrm{Fe}^{-}$by Gurimskaya et al. [17] Lauwaert et al. [13] have led to the following trapping parameters: $\sigma_{\infty}=3.7 \times 10^{-15} \mathrm{~cm}^{2} \quad \Delta E_{\sigma}=50 \mathrm{meV}[13]$ $\sigma_{\infty}=4.98 \times 10^{-15} \mathrm{~cm}^{2} \quad \Delta E_{\sigma}=43 \mathrm{meV}[17]$ from which at $\mathrm{T}=155 \mathrm{~K} c_{n} n=6 \times 10^{4} s^{-1}$ is calculated.

The experimental $\Delta C\left(t_{p}\right)$ and $\Delta C\left(t_{p}\right) / C_{r}^{3}$ curves are shown in Fig. 7 and the analysis 
results, as a function of the first $t_{p}$ data point included in the analysis, are summarized in Fig. 8. A good qualitative similarity between Fig. 8 and Fig. 6 is noted: Method 1 leads to slightly lower capture rates than the Analytical Model, and Method 2 to slightly higher results. Methods 1 and 2 again exhibit a rather sharp transition range in which the fitting becomes unreliable situated at $10 \mu s<t_{p}<30 \mu s$. When using the Analytical Method, the fitting result remains nearly unaffected up to $t_{p}=100 \mu \mathrm{s}$, which implies a significant extension of the range of measurable capture rates and cross-sections. Like in the simulation example, the fitting results for Method 1 and 2 are affected by the $V_{r}$ value at (or up to) which the data were recorded and results in somewhat closer agreement with one another are found for the largest $\left|V_{r}\right|$ value. However, there is an important qualitative difference with the simulated data: all fitting methods yield a larger capture rate for the highest $\left|V_{r}\right|$ value. Although this might be a consequence of fitting real experimental data for samples which, e.g., may exhibit (small) majority carrier density profiles that influence fitting results, it might also point to an electric field dependence of the electron capture cross-section.

\section{MEASUREMENTS PERTURBED BY AN ADDITIONAL TIME CONSTANT}

Pons already noticed that as particular consequence of Eq. (1), for a given pulse duration $t_{p}$ there exists a threshold pulse amplitude $\left|V_{p}-V_{r}\right|$ for obtaining a measurable DLTS amplitude $\Delta C$ [8]. The $t_{p}$ dependence of $L_{p}$ may, indeed, induce a dead time in the $\Delta C\left(t_{p}\right)$ curve where the signal remains zero. In Figs. 2 and 7 , the curves at $V_{r}=-0.25 V$ exhibit such behavior. A similar dead time may also be provoked by other effects, e.g. additional potential barriers (next to the main junction diode). In this case the $\Delta C\left(t_{p}\right)$ cannot even be modeled by numerically solving Eq. (1), because the cause for the dead time lies outside the model. In order to test which fitting method is most suitable for analyzing the capture kinetics in such case, an additional time constant $\tau=1 \times 10^{-7} s$ was introduced in the simulated data of Figs. 2 and 3 by allowing the potential drop over the junction diode to vary like

$$
\Delta V(t)=\left|V_{p}-V_{r}\right|\left(1-e^{-\frac{t}{\tau}}\right)
$$

Figure 9 shows the simulated data at $V_{r}=-5 \mathrm{~V}$ along with the best fit with the Analytical Model. Not only is the shape of the $\Delta C\left(t_{p}\right)$ curve only poorly reproduced, the capture rate 


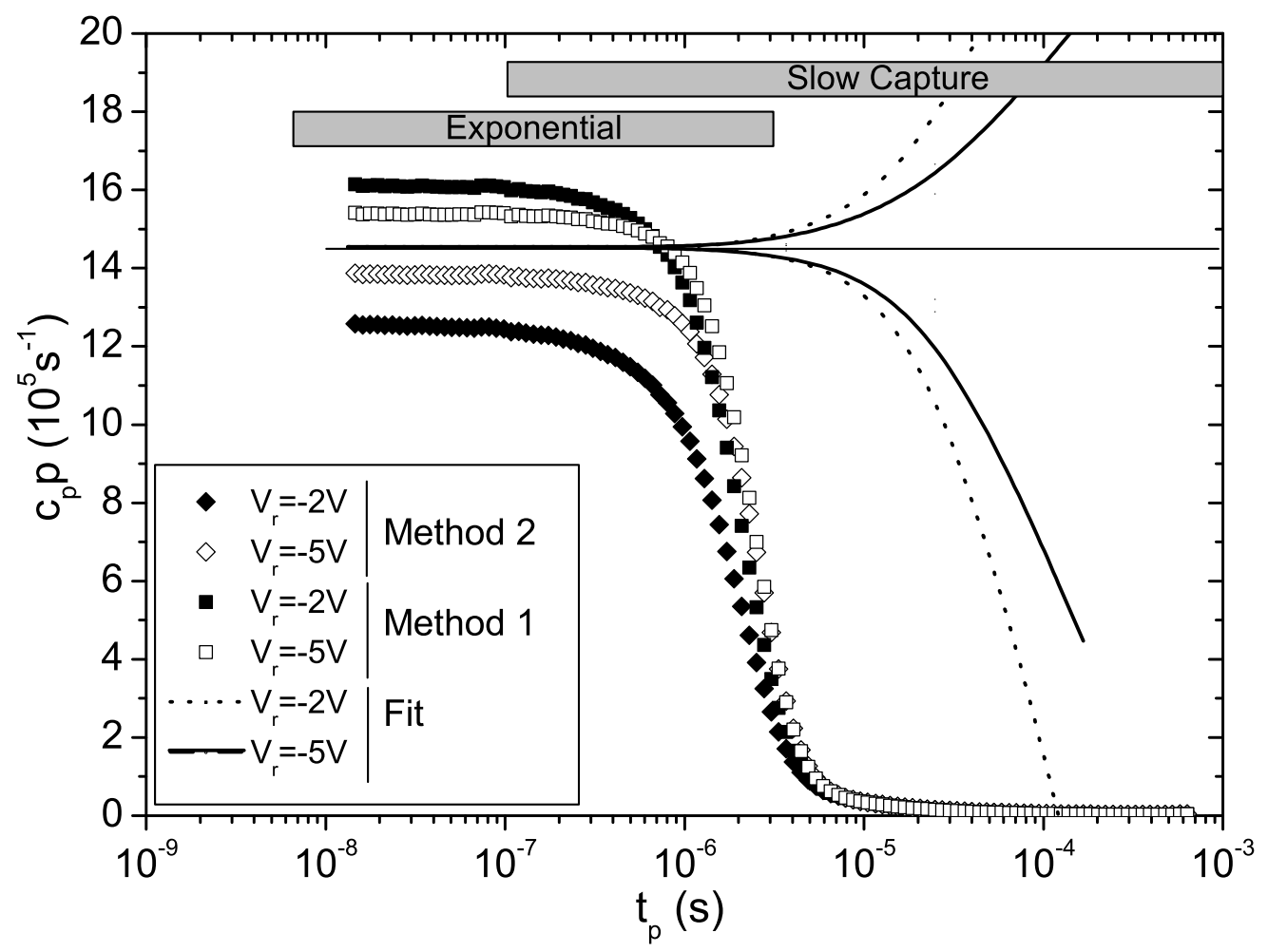

FIG. 6: Estimated capture rate as a function of $t_{p}$ shortest pulse length available for the simulated data. For the Analytic Model the lines correpond with the borders of the $95 \%$ confidence interval.

$c_{p} p$ is also underestimated by about $40 \%$. Figure 10 represents the $\ln \left(1-\Delta C\left(t_{p}\right) / \Delta C_{\infty}\right)$ curves at different $V_{r}$. They all show a linear dependency, but this lines do not pass through the origin, however. This presents a complication in the application of Method 1 and obviously leads to underestimations of $c_{p} p$. For Method 2, one can without any problem determine the slope of each curve in Fig. 10 at the $t_{p}$ point where it reaches a maximum and find $c_{p} p$ by extrapolation to $C_{r}^{2} \rightarrow 0$. In an analogous fashion as Figs. 6 and 8, Fig. 11 summarizes the analysis results of the data including an extra time constant following the (three) methods discussed in this work. When data are available from sufficiently small $t_{p}$ onwards, Method 2 clearly emerges as the superior method, overestimating the capture rate used for generating the data points only very slightly. Method 1 and the Analytical Method perform significantly poorer, both underestimating the capture rate considerably. 

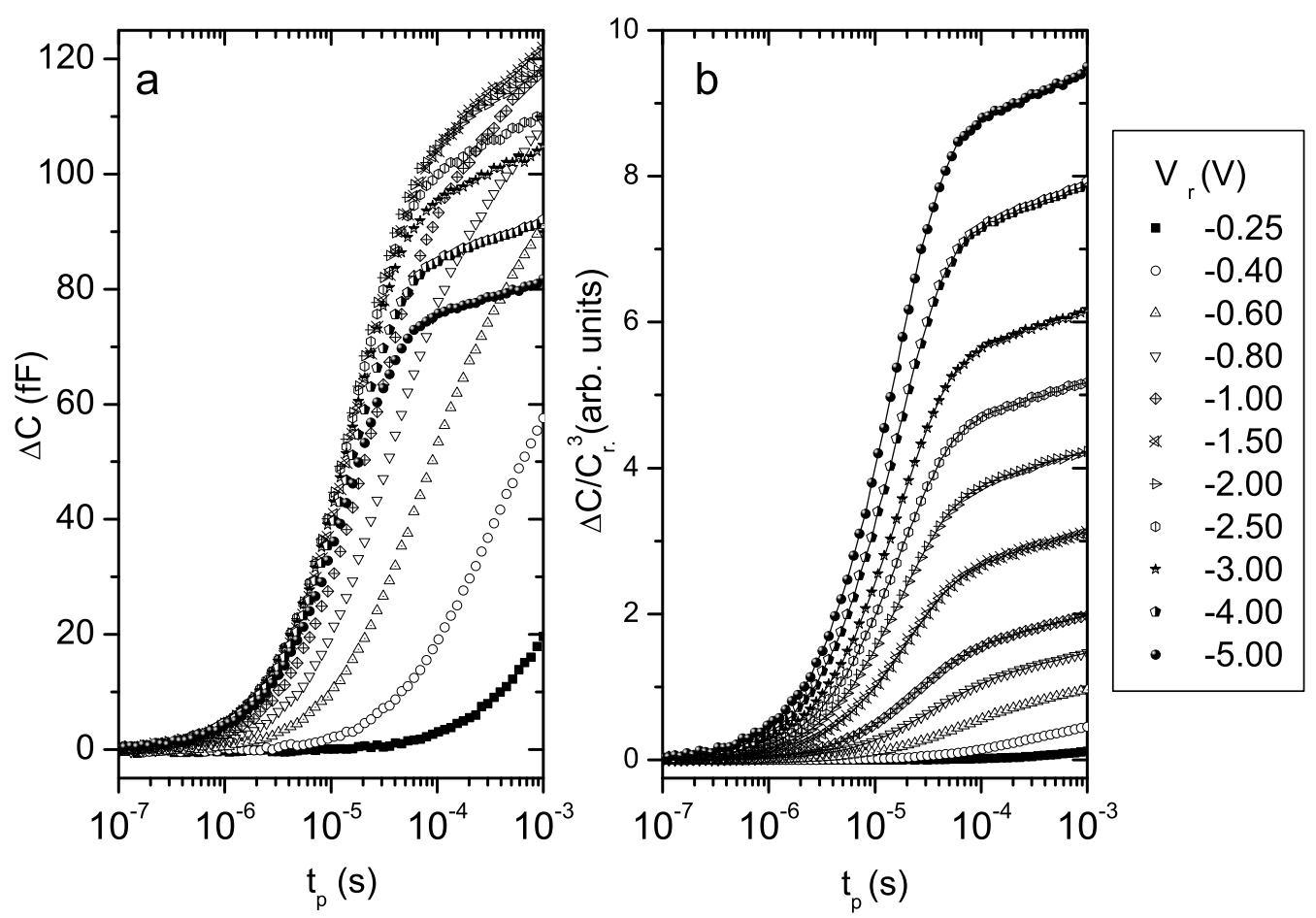

FIG. 7: (a) DLTS signal amplitude as a function of filling pulse duration $t_{p}$ recorded for the $F e^{-/ 2-}$ level at $155 \mathrm{~K}$. (b) Data corrected for $C_{r}^{3}$.

\section{CONCLUSIONS}

Obtaining accurate values of the majority carrier capture rates and cross-sections for deep-level defects from DLTS experiments is not so straightforward as it may seem at first glance. Without detailed knowledge of the entropy changes in capture and emission, direct calculation from the pre-exponential factor in the Arrhenius analysis of the emission rates may at best yield the right order of magnitude. Analysis of isothermal pulse length variation experiments can only yield good estimates of capture cross-sections if the $\Delta C\left(t_{p}\right)$ curves are not completely dominated by slow capture effects and not too strongly affected by additional time constants in the experimental devices, which delay the growth of $\Delta C$. From the experimental side, it is certainly recommended to record $\Delta C\left(t_{p}\right)$ data at various reverse voltage values: inspecting the resulting changes in the curves allows to identify the problems that will occur in the analysis. The simple approach of Method 1, which ignores the influence 


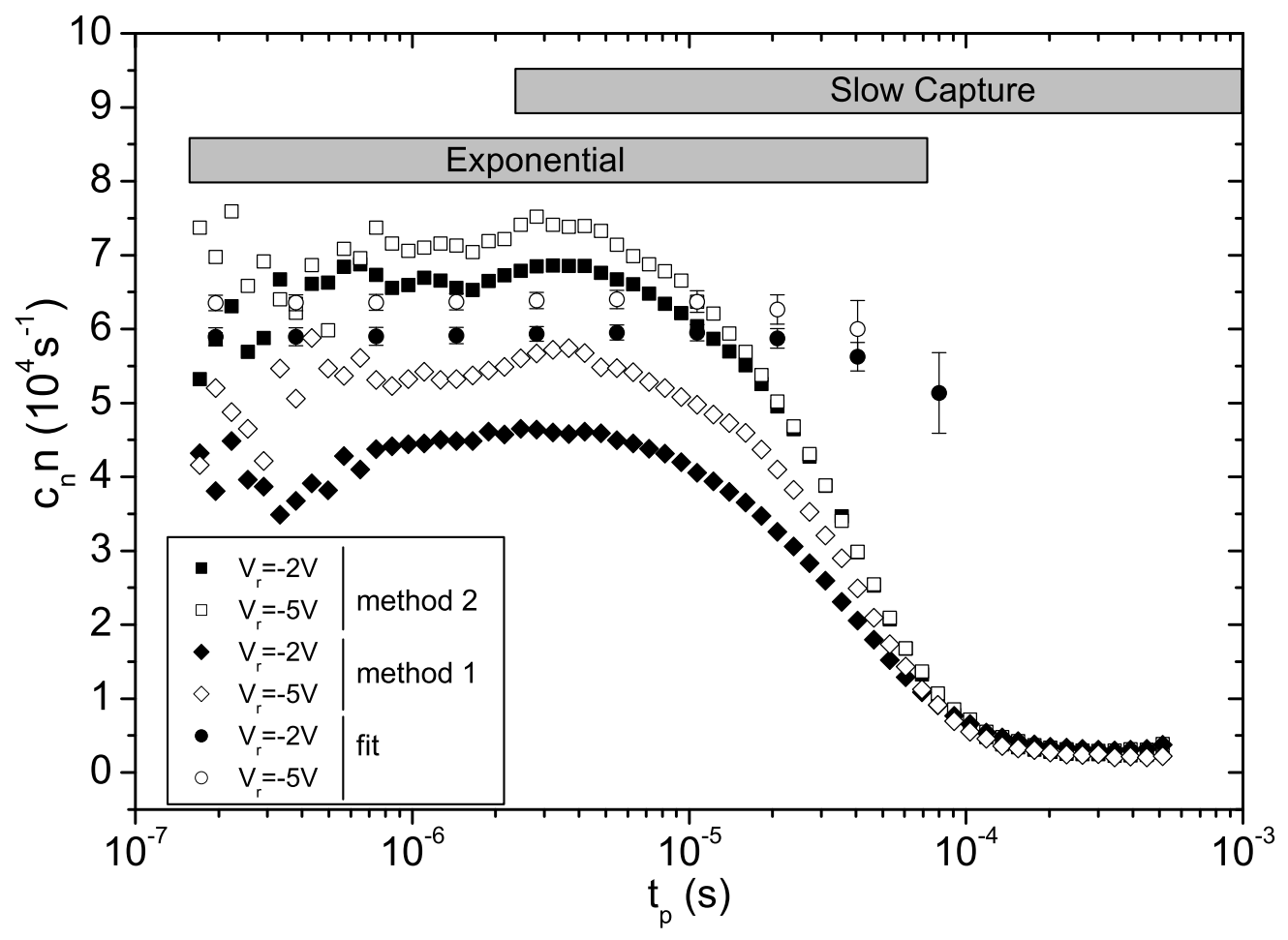

FIG. 8: Estimated capture rate as a function of $t_{p}$ shortest pulse length available for electron capture by the $\mathrm{Fe}^{-/ 2-}$ level.

of additional time constants and aims to avoid the range where slow capture effects affect the data, necessarily leads to underestimates of the cross-section. If the $\Delta C\left(t_{p}\right)$ curves are mainly distorted at large tp by slow capture (and not so much by delayed growth at small $t_{p}$ ), fitting the data with the Analytical Model definitely presents the most accurate and most stable solution. On the other hand, if the $\Delta C\left(t_{p}\right)$ curves are notably affected by an additional time constant at small $t_{p}$, this method may also lead to considerable underestimates of the capture cross-section. In such case, Method 2, which uses maximum slope analysis of the $\Delta C\left(t_{p}\right)$ curves and extrapolation of the $V_{r}$ dependence of the results, may present the best solution. 


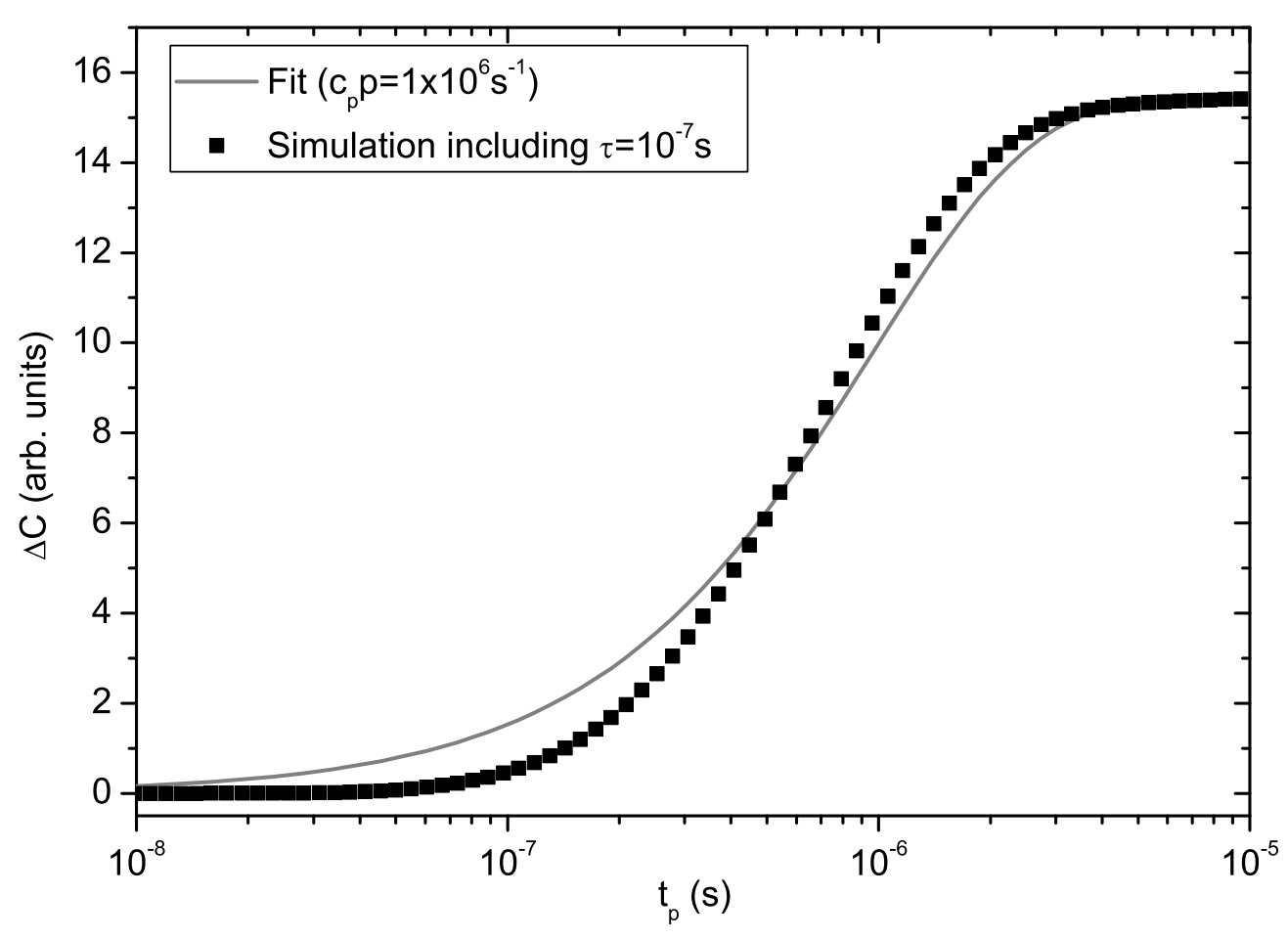

FIG. 9: Simulated DLTS signal amplitude using the parameters in Table I and an additional time constant. The best fit using the Analytic Model is also inculded in the graph.

\section{Acknowledgments}

S. Khelifi wishes to acknowledge the financial support of the H2020 project SWInG (Grant agreement 640868) sponsored by The European Research Council. H. Vrielinck whishes to acknowledge the FWO for financial support through grant $\mathrm{n}^{\circ}$ G.0207.10N.

[1] D.V. Lang, J. Appl. Phys. 45, 3023 (1974)

[2] S. Weiss and R. Kassing, Solid St. Electron. 31,1733 (1988)

[3] L. Dobaczewski, A. R. Peaker and K. Bonde Nielsen, J. Appl. Phys., 96, 4689 (2004)

[4] A.R. Peaker, V.P. Markevich, I.D. Hawkins, B. Hamilton, K. Bonde Nielsen, K. Goscinski, Physica B 407, 3026 (2012) 


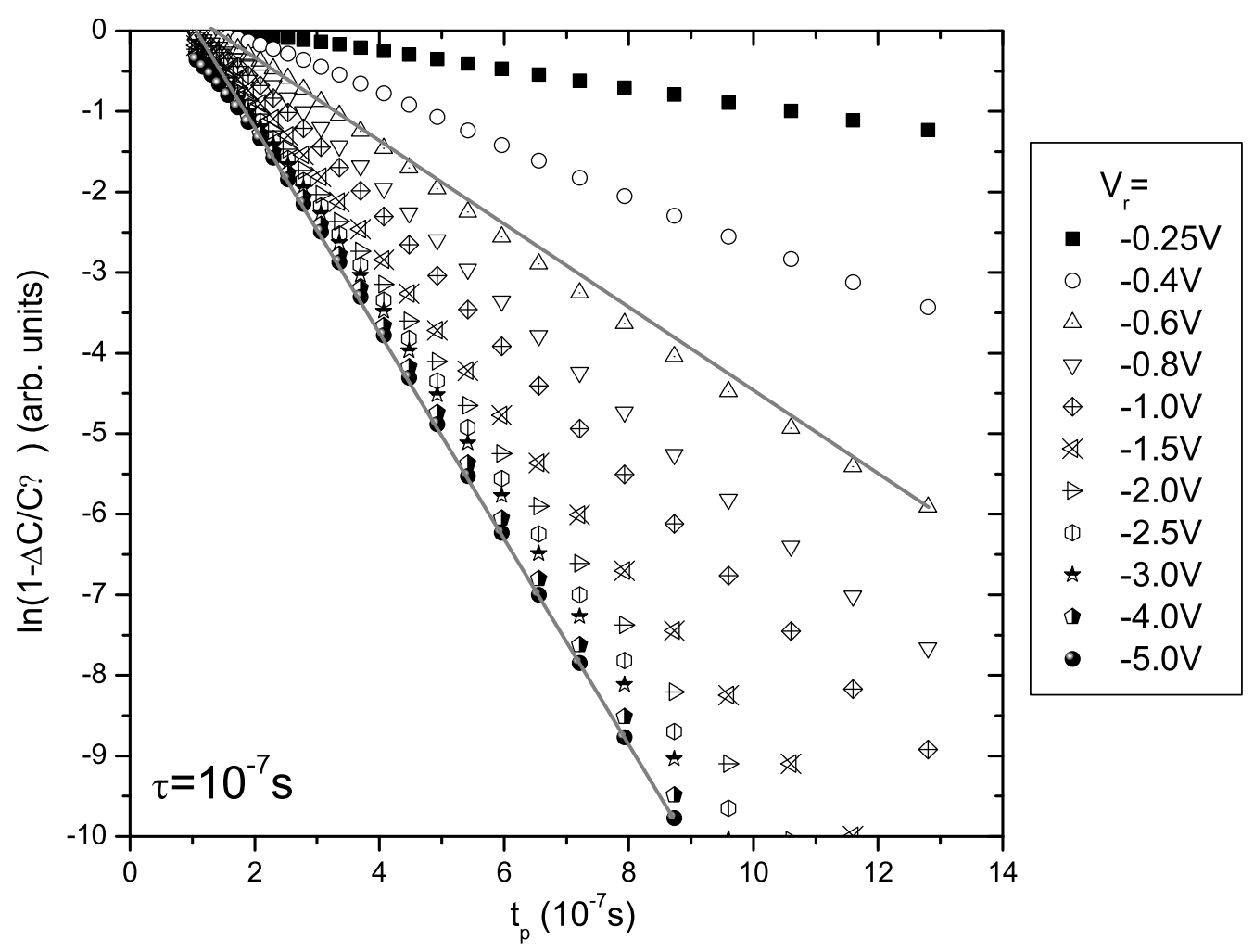

FIG. 10: $\ln \left(1-\frac{\Delta C\left(t_{p}\right)}{\Delta C_{\infty}}\right)$ for the calculated DLTS-signal amplitudes as a function of filling pulse duration for the data simulated including a time constant.

[5] J. Lauwaert, L. Callens, S. Khelifi, K. Decock, M. Burgelman, A. Chirila, F. Pianezzi, S. Buecheler, A. N. Tiwari and H. Vrielinck, Prog. Photovolt: Res. Appl. 20(5), 588594 (2012)

[6] J. Lauwaert, S. Khelifi, K. Decock, M. Burgelman and H. Vrielinck, J. Appl. Phys. 109, $063721(2011)$

[7] E. Simoen, J. Lauwaert and H. Vrielinck, Chapter 6 Analytical Techniques for Electrically Active Defect Detection in Semiconductors and SemiMetals Volume 91 Editors L. Romano, V. Privitera and C. Jagadish

[8] D. Pons, J. Appl. Phys. 55, 3644 (1984)

[9] J. Lauwaert, J. Van Gheluwe and P. Clauws, Rev. Sci. Instrum. 79, 093902 (2008)

[10] P. Blood and J.W. Orton, 'The electrical characterization of semiconductors: majority carriers and electron states', Academic Press (1992). 


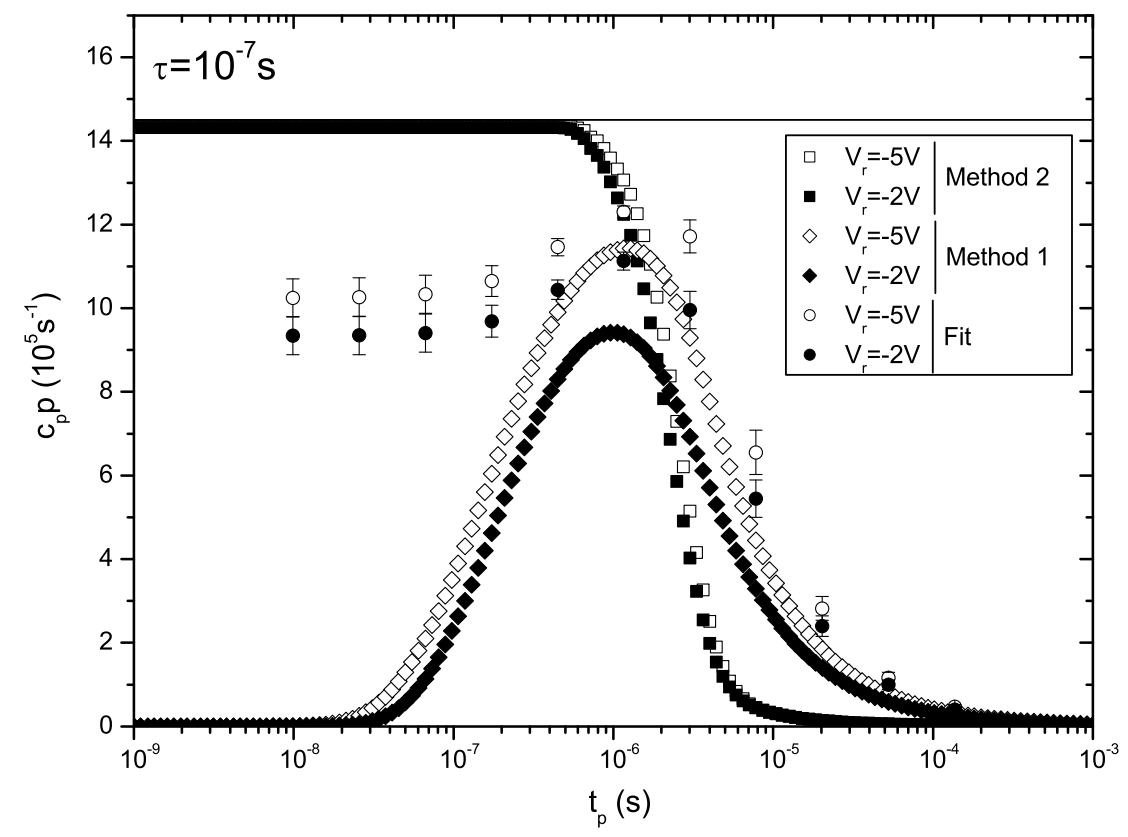

FIG. 11: Estimated capture rate as a function of $t_{p}$ shortest pulse length available for the simulated data including a time constant $\tau=10^{-7} \mathrm{~s}$.

[11] P. Clauws, J. Van Gheluwe, J. Lauwaert, E. Simoen, J. Vanhellemont, M. Meuris, and A. Theuwis, Physica B 401402, 188 (2007)

[12] J. Lauwaert, J. Van Gheluwe, J. Vanhellemont, E. Simoen and P. Clauws, J. Appl. Phys. 105 $073707(2009)$

[13] J. Lauwaert, J. Vanhellemont,E. Simoen, H. Vrielinck and P. Clauws, J. Appl. Phys. 111 $113713(2012)$

[14] J. Lauwaert, S.H. Segers, F. Moens, K. Opsomer, P. Clauws, F. Callens, E. Simoen and H. Vrielinck, J. Phys. D: Appl. Phys. 48175101 (2015)

[15] J. Lauwaert and P. Clauws, Thin Solid Films 518, 2330 (2010)

[16] S.H. Segers, J. Lauwaert, P. Clauws, E. Simoen, J. Vanhellemont , F. Callens and H. Vrielinck, Semicond. Sci. Technol. 29125007 (2014)

[17] Y. Gurimskaya, D. Mathiot, A. Sellai, P. Kruszewski, L. Dobaczewski, A. Nylandsted Larsen and A. Mesli, J. Appl. Phys. 110 , 113707 (2011) 